lich differenzierte Betrachtung verdeutlicht jedoch die Konzentration von Kriminalität und sozialen Problemen in den Blöcken der Mittelachse (ebd.: 327). Die hier herrschende Atmosphäre strahlte mit hoher Wahrscheinlichkeit auf die ganze Siedlung aus.

10 In den 80er Jahren stellten Studien fest, dass insbesondere männliche Jugendliche entwicklungsbedingt hin und wieder kleinere Delikte begehen, nur selten erwischt werden und im Allgemeinen von alleine wieder aufhören, sowie sie älter geworden sind (vgl. Sessar 1984).

11 Die hohe Wohndichte bewirkt »dass Menschen mehr Zeit außerhalb ihrer zu kleinen Wohnungen verbringen, ihre Kinder weniger überwachen und das Konfliktpotential innerhalb der Familien höher ist « (Hermann / Laue 2001: 100).

12 Die Bezeichnung ,Sanierung‘ wird im Folgenden beibehalten, obwohl die (sich über einen Zeitraum von 10 Jahren und länger erstreckenden) Maßnahmen zu Programmen der Sanierung, Revitalisierung und ,Stadterneuerung in kleinen Schritten' (SteP) gehörten.

13 Vgl. zur Einbettung der Zahlen in die gesamtstädtische Entwicklung Sessar/Herrmann/Keller/Weinrich / Breckner 2003.

14 Der seit den 90er Jahren im Verlauf der ökonomischen Krise einsetzende Rückzug des Sozialstaates aus verschiedensten Leistungen bedeutete auch einen
Rückzug aus dem sozialen Wohnungsbau. Es wurden kaum bis keine neuen Bestände geschaffen und ehemalige Sozialwohnungen aus den Mietpreis-Bindungen genommen oder privatisiert, so dass weitere Investitionen möglich wurden und die Mieten stiegen. Diese Liberalisierung des Wohnungsmarktes führte zu einer weiteren Konzentration des Bestandes in den Gebieten, in denen dieser Mechanismus nicht einsetzte. 15 Statistiken hierzu liegen noch nicht vor.

16 Die Ergebnisse beziehen sich zum überwiegenden Teil auf die Großwohnsiedlung; ein kleiner Teil der Befragten sind Bewohner eines angrenzenden Stadtteils sowie ,Alt-Steislhooper', da das die Grenzen des Untersuchungsgebiets über die eigentliche Großwohnsiedlung hinaus gingen.

17 Bereits im Dezember 2002 titelte die Zeitung die WELT: "Geldmangel: Sozialprojekten in Steilshoop droht das Aus « (20.12.2002).

18 Das durch die damalige Stadtentwicklungsbehörde herausgegebene, sich $u$. a. auf das Sanierungsgebiet Steilshoop beziehende Konzept ,Stadterneuerung Hamburg' (1996) ließt sich heute wie ein Vorläufer der Sozialen Stadtentwicklung (s. u.).

19 Das Hamburger Programm wurde später als ,Programm sozialer Stadtentwicklung' weitergeführt (vgl. hierzu den Bericht von Mayer 2004).
20 So auch die Charakterisierung von Stangl für Wien (2005: 11).

21 So wurde etwa auch die Stadtentwicklungsbehörde als Fachbehörde (vergleichbar den Ministerien anderer Bundesländer) aufgelöst und die Zuständigkeit der Fachbehördenebene insgesamt neu sortiert.

22 Dieser Optimismus ließe sich in Richtung einer bestehenden, von vielen Schultern gestützten »culture of control« (Garland 2001) interpretieren, die im Großen und Ganzen den politischen und gesellschaftlichen Veränderungen in der Stadt standhält. Die Zukunft wird erst zeigen, ob die Sparmaßnahmen in den verschiedensten Bereichen nicht doch zu einem erneuten Umkippen der (für die Enge und Struktur der Großwohnsiedlung erstaunlich) friedlichen Situation führen können.

23 Unter dem ,ganzheitlichen Ansatz' verstehen Feltes und Trenczek / Pfeiffer dagegen die umfassende Sicht auf die Probleme.

24 Der Ministerialdirigent Jörg Ziercke (Leiter der Polizeiabteilung im Innenministerium Schleswig-Holstein) schlägt die Entwicklung eines Berufsbildes des ,kommunalen Präventionsmanagers' vor (Ziercke 2001: 4).

\title{
Wie oft ängstigen sich Ängstliche, wenn sie sich ängstigen? Häufigkeit und Intensität von Krimina- litätsängsten am Beispiel der Wiener Bevölkerung
}

Gert Feistritzer und Wolfgang Stangl

\begin{abstract}
- mpirische Studien zu Fragen der Kriminali-
- tätsängste nationaler oder städtischer Bevölkerungen gehören $\mathrm{zu}$ den häufigsten Themen, mit denen sich die Kriminalsoziologie in den letzten rund 20 Jahren in Europa und den USA beschäftigt hat. Vielfach verbinden sich derartige Studien mit Fragen nach Möglichkeiten, wie künftig mit Kriminalitätsängsten umzugehen sei, oder auf welche Weise man sie verhindern könne, womit das zweite große kriminalsoziologische Forschungsthema angesprochen ist: Die Kriminalprävention in ihren verschiedenen ideologischen und thematischen Bezügen.
\end{abstract}

Die Zusammenführung beider Themen auf der Ebene der jeweils nationalen, aber auch der europäischen Justiz- und Innenpolitik führte zur Herausbildung des kriminalpolitischen "Superthemas «, dem deswegen so große Bedeutung zukommt, weil es aus dem engeren "Sachbereich « herausgetreten ist und sich an viele andere Sachthemen angelagert hat: Wir meinen das Thema der »Inneren Sicherheit « (vgl. Stangl 2003). Es ist dies mittlerweile ein politisches, ökonomisches wie auch wissenschaftliches Feld, auf dem die unterschiedlichsten Interessengemeinschaften, Perspektiven und Akteure ihren Platz finden und

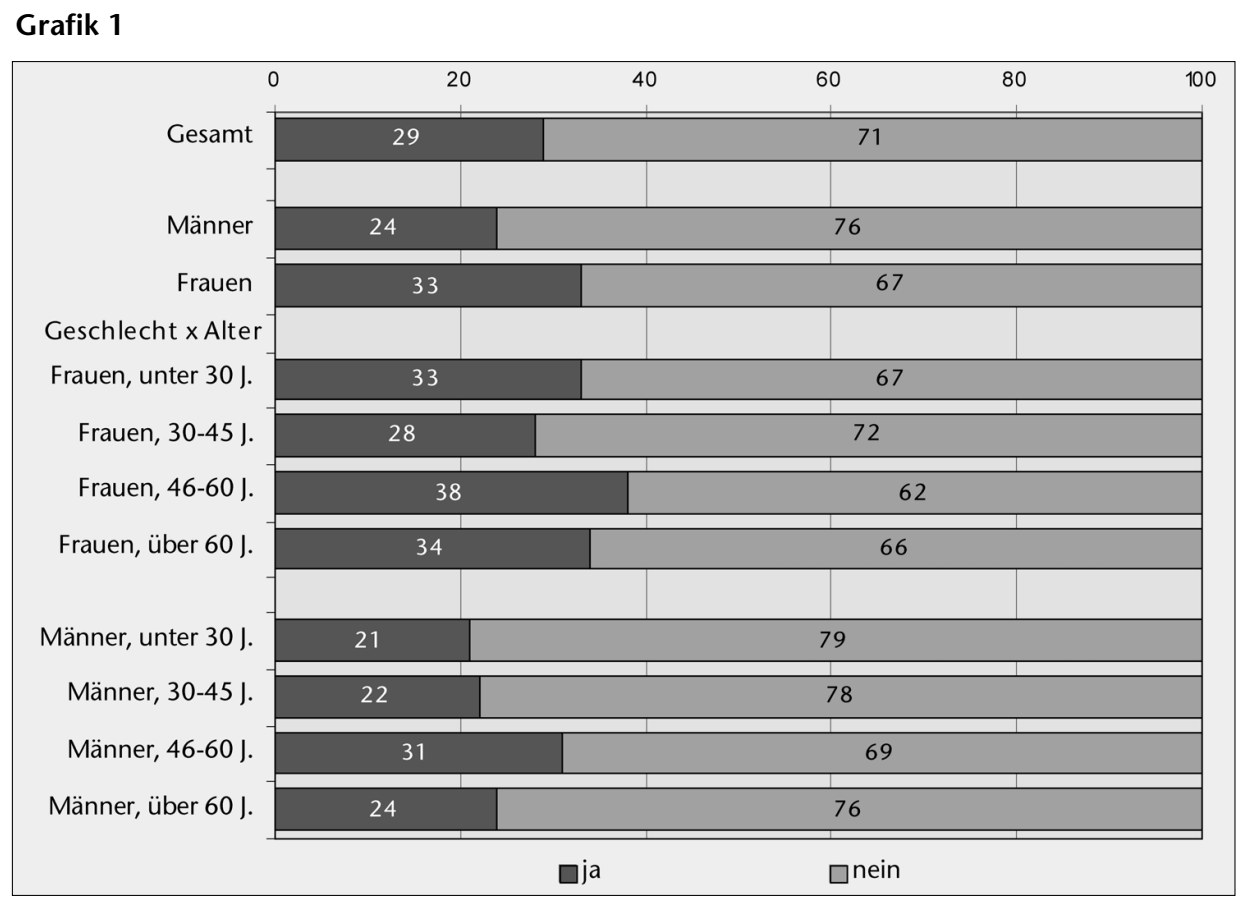

Frage: Haben Sie im letzten Jahr einmal Angst gehabt, in Wien Opfer einer Straftat zu werden? (in Prozent) 
Grafik 2

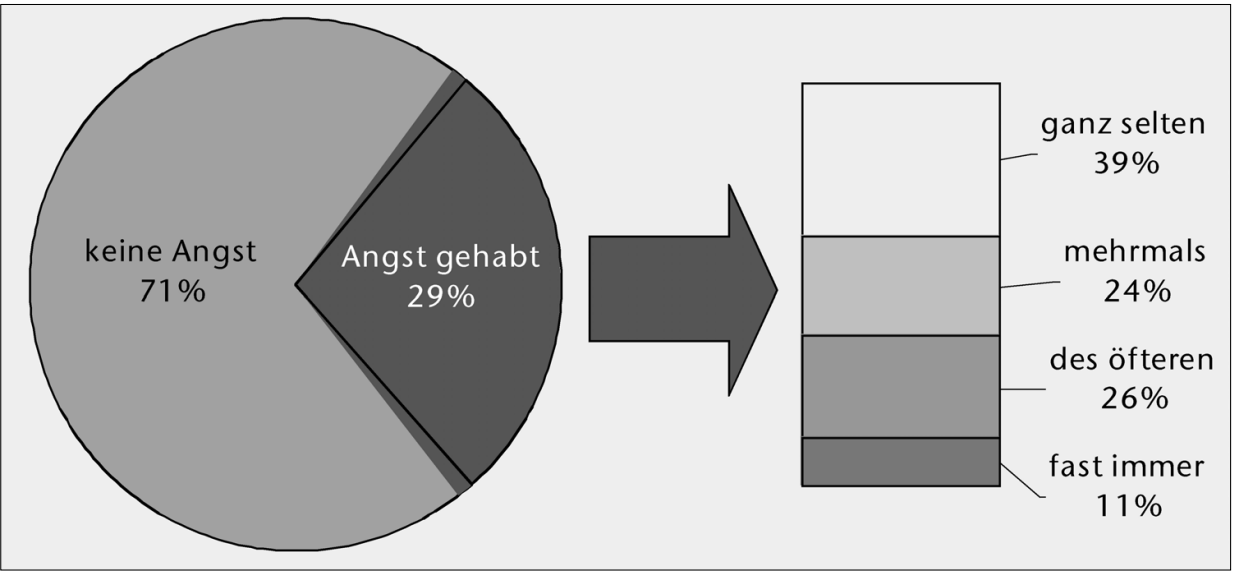

Frage: Haben Sie im letzten Jahr einmal Angst gehabt, in Wien Opfer einer Straftat zu werden? Wenn ja: Wie häufig...?

tätig werden und damit den Umfang wie auch Inhalt des Begriffs »Sicherheit« weiter entwickeln (Bauman 2005, 90-132).

Aus Sicht der Wissenschaft ist erstaunlich, dass angesichts der angesprochenen Bedeutung der Forschungen zu Kriminalitätsängsten, Fragen nach der Häufigkeit wie auch Intensität dieser Ängste bisher kaum geprüft worden sind. Ditton und Farrall (2000) haben darauf hingewiesen, dass zahlreiche Studien im anglo-amerikanischen Raum der 1965 im Auftrag der Presidential Commission on Law Enforcement and Administration of Justice (Biderman et.al. 1967) durchgeführten Forschung gefolgt sind, in der erstmals die große Bedeutung der Kriminalitätsfurcht erheblicher Teile der Bevölkerung doku-

\section{Grafik 3}

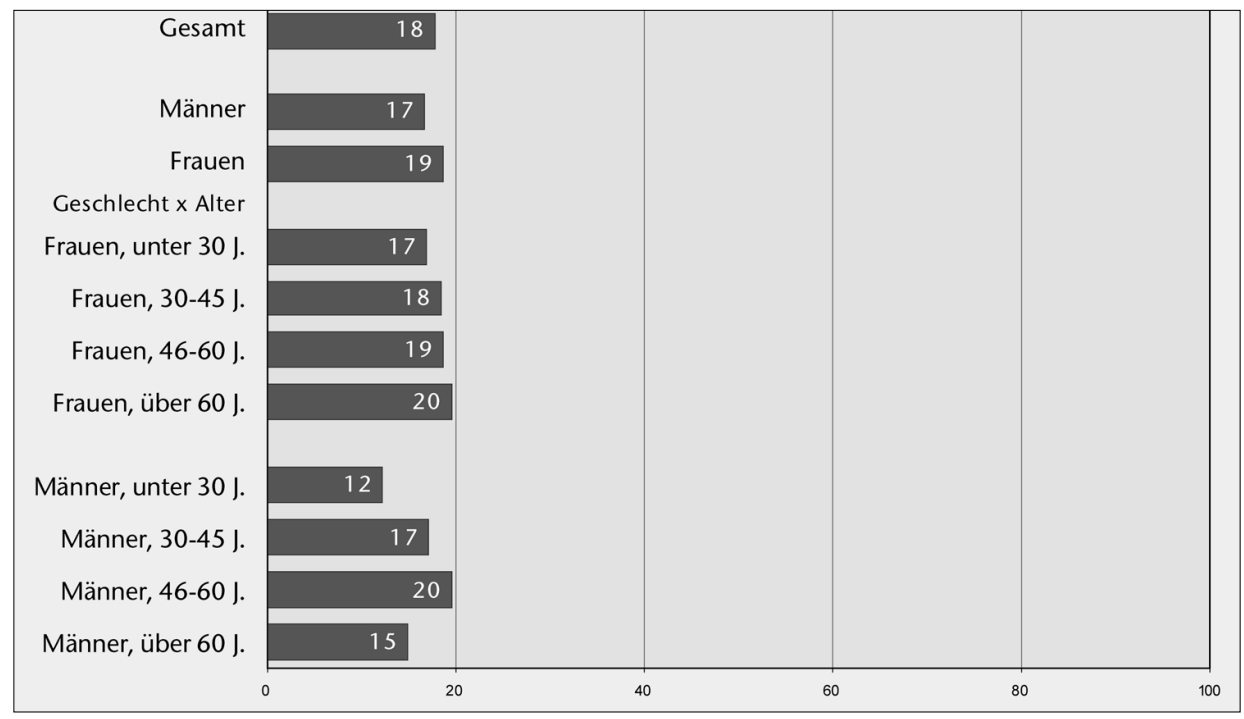

Frage: Wie häufig haben Sie in den letzten 12 Monaten Angst gehabt, in Wien Opfer einer Straftat zu werden?

(in Prozent; Basis: Gesamtbevölkerung; mehrmals / des öfteren / fast immer)
1. "Haben Sie im letzten Jahr einmal Angst gehabt Opfer einer Straftat zu werden? «

2. "Wie häufig haben Sie Angst gehabt, Opfer einer Straftat zu werden? « (Diese Frage wurde nur an jene gerichtet, die Frage $1 \mathrm{mit} » j \mathrm{j}$ « beantwortet hatten)

3. »Wie stark war Ihre Angst, als Sie sich das letzte Mal gefürchtet hatten?

Die Befragung brachte folgende Hauptergebnisse:

\section{»Habe Angst gehabt «}

Insgesamt $29 \%$ der WienerInnen haben im vergangenen Jahr (März 2004 bis März 2005) befürchtet, in Wien Opfer einer Straftat zu werden (vgl. Grafik 1). Dass dieser Anteil »Ängstlicher « im internationalen Vergleich niedrig ist, sei hier unterstrichen, soll aber nicht weiter begründet werden*. Für die Untersuchung der Frage nach Häufigkeit und Intensität von Kriminalitätsängsten ist die grundsätzliche Verteilung dieser Angst in der Stadtbevölkerung unerheblich.

Während die Angst, Opfer einer Straftat zu werden, auf ein Drittel der Wiener Frauen zutrifft, liegt der entsprechende Anteil bei den Männern bei knapp einem Viertel. Dieser Unterschied zwischen den Geschlechtern, der sich tendenziell in allen Altersgruppen abbildet, ist im internationalen Vergleich als eher gering $\mathrm{zu}$ qualifizieren. Bemerkenswert ist, dass sowohl bei den Männern wie bei den Frauen die vergleichsweise höchsten Nennungsanteile bei der Gruppe der 46- bis 60-Jährigen ausgewiesen sind; also nicht bei den untersten Altersstufen und auch nicht bei den älteren Leuten (dies indiziert, dass sich die Befürchtungen wohl primär auf mögliche Eigentumsdelikte beziehen, etwa auf einen Autodiebstahl oder Ähnliches).

\section{Die Häufigkeit der Kriminalitätsängste}

Von jenen, die im Laufe der letzten 12 Monate Ängste vor einer Straftat hatten (das sind 29\%), sagten vier von zehn Befragten, dass dies nur einmal oder ganz selten der Fall gewesen sei (vgl. Grafik 2). Bei jeweils einem Viertel der von diesem Gefühl Tangierten traf dies mehrmals oder öfters zu. 11 Prozent dieser Gruppe leben unter fast ständiger Furcht. Umgerechnet auf die Gesamtbevölkerung umfassen die Letztgenannten (»Fürchte mich fast immer«) 3 Prozent. Rechnet man auch jene hinzu, die mehrmals oder öfters Ängste gehabt haben, so kommt man auf einen Gesamtanteil von 18 Prozent an BewohnerInnen, die nicht in einer weitgehenden Unbesorgtheit vor Viktimisierung leben.

Knapp eine Fünftel der Bevölkerung hatte im letzten Jahr »mehrfach « bis »fast immer « die Befürchtung, Opfer eines Deliktes werden zu können. Bei diesen »Ängstlichen « ist wiederum bemerkenswert, dass es hier kaum Unterschiede 


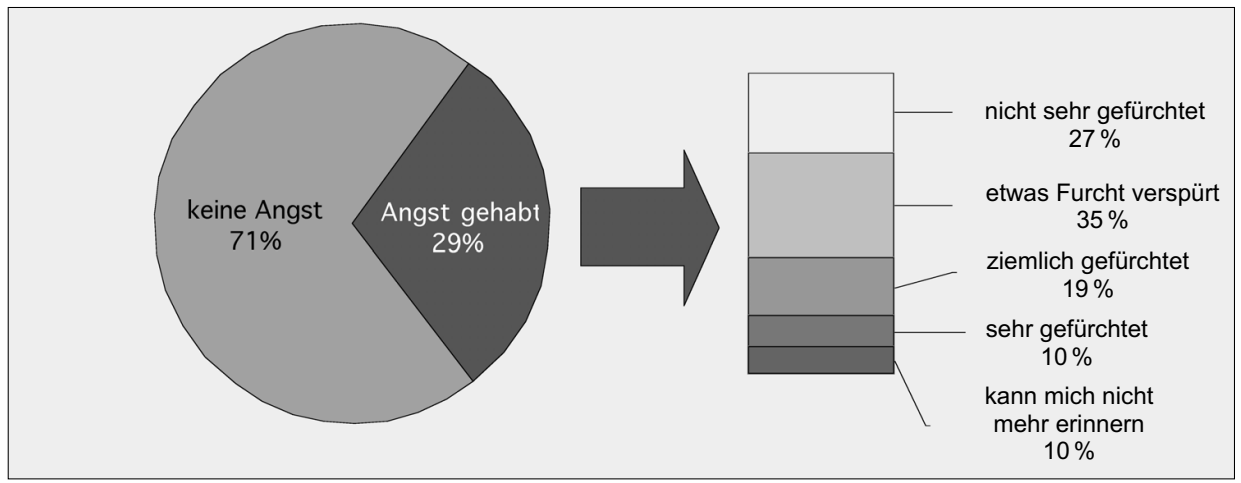

Frage: Haben Sie im letzten Jahr einmal Angst gehabt, in Wien Opfer einer Straftat zu werden? Wenn ja: Wie stark war Ihre Angst...?

\section{Grafik 5}

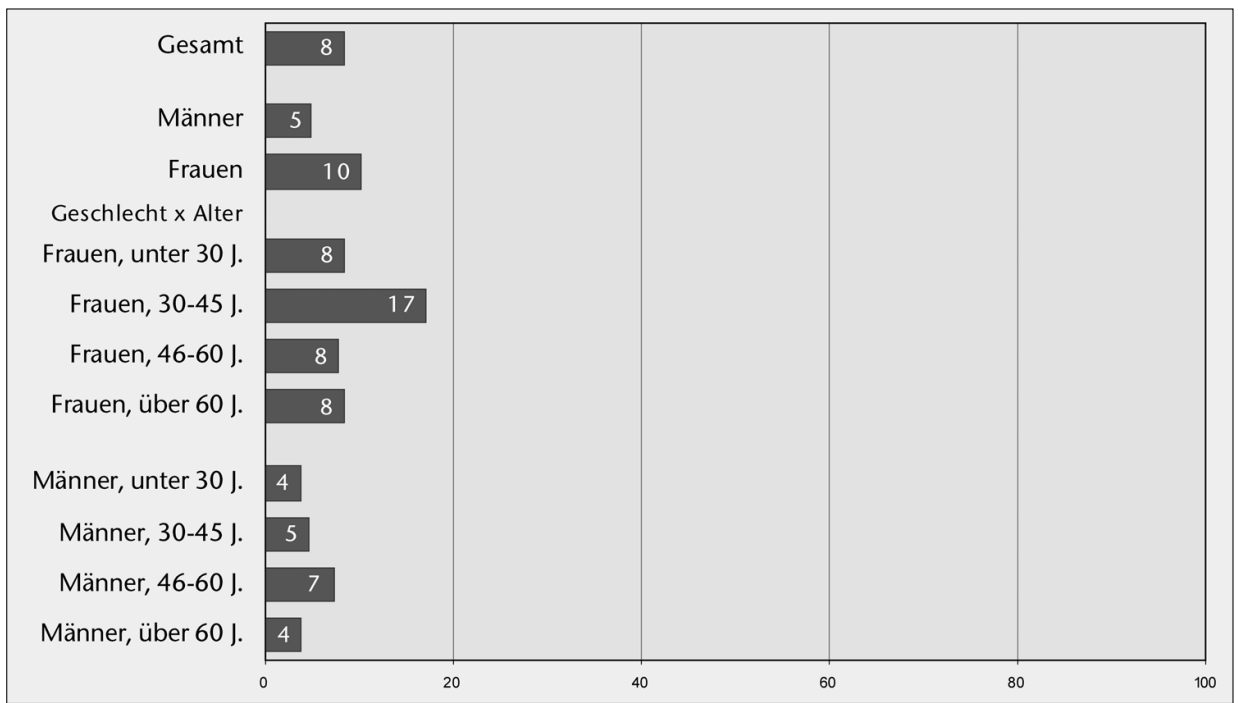

Frage: Wie stark war Ihre Angst, als Sie sich das letzte Mal in Wien vor einer Straftat gefürchtet haben?

(in Prozent; Basis: Gesamtbevölkerung; »habe mich ziemlich / sehr gefürchtet «)

zwischen den Frauen und den Männern gibt (vgl. Grafik 3). Leichte Abweichungen manifestieren sich nur insofern, als die Nennungsanteile innerhalb der Gruppe der Männer bei den mittleren Altersgruppen wieder etwas erhöht ausfallen, während diesbezüglich zwischen den Altersgruppen der Frauen so gut wie keine Abweichungen aufscheinen.

\section{Intensität der Kriminalitätsängste}

Wie stark bzw. in welcher Intensität werden nun die Ängste vor Delikten in Wien verspürt? Von jenen, die im letzten Jahr zumindest einmal ein Unsicherheitsgefühl hatten, gaben 27 Prozent an, dass sie sich dabei bzw. beim letzten diesbezüglichen Vorfall nicht sehr gefürchtet haben. Weitere 9 Prozent konnten sich an das Ausmaß ihrer Furcht nicht mehr entsinnen, woraus sich wohl schließen lässt, dass dasselbe nicht allzu groß sein konnte. In Summe haben also 36 Prozent offenkundig nur sehr geringe Ängste empfunden; rund ebenso viele (35\%) gaben an, etwas Furcht verspürt zu haben. Weiters haben sich 19 Prozent der »Ängstlichen« »ziemlich « und 10 Prozent »sehr « geängstigt. Also bei knapp drei von zehn Betroffenen war dies in stärkerem Maße der Fall. Umgerechnet auf die Gesamtbevölkerung beschränken sich die intensiveren Angsterlebnisse auf 8 Prozent der Befragten. Innerhalb dieser Gruppe ist der Anteil der Frauen mit 10 Prozent doppelt so hoch wie jener der Männer.

\section{Intensität und Häufigkeit der Angst}

Von der relativ kleinen Gruppe derer, die angab, sich oft vor Kriminalität zu fürchten, gab nur eine Minderheit von 15 Prozent zu Protokoll, dabei zuletzt große Ängste verspürt zu haben. Demgegenüber sagten 57 Prozent, dass dieselben eher gering waren. Noch deutlich abgeschwächter fiel die Furchtintensität bei jenen aus, die hin und wieder Angst vor einem Kriminalitätsdelikt verspüren. Bei den Personen, bei welchen das ganz selten der Fall war, beschränkten sich die starken Ängste auf 6 Prozent, während knapp zwei Drittel eher nur ein Unwohlgefühl verspürten. Ähnlich lautet die Verteilung bei der Gruppe, die angab, im letzten Jahr mehrmals Angst vor Kriminalität gehabt zu haben: 8 Prozent empfanden dabei starke Ängste, bei knapp drei Viertel fielen dieselben ihren Angaben zufolge eher moderat aus.

Bezogen auf die Gesamtbevölkerung ergibt sich folgende Verteilung: Eine breite Mehrheit von 71 Prozent hat keine Kriminalitätsängste. Bei der Restgruppe überwiegen, weitgehend unabhängig davon, wie häufig sie im letzten Jahr Angst vor Kriminalität gehabt haben, jeweils jene, die dabei von einer eher geringen Furchtintensität sprechen. Es handelt sich dabei, je nach Häufigkeit der Angsterlebnisse, um 5 bis 7 Prozent der Wienerinnen und Wiener (in Summe um $18 \%)$.

Deutlich geringer sind die Quoten derer, die angaben, sich ziemlich oder sehr gefürchtet zu haben. Nur jeweils 2 Prozent der Bevölkerung hatten im letzten Jahr selten oder mehrmals Angst vor Kriminaldelikten und sich dabei anlassbezogen ziemlich oder sehr gefürchtet. Mit insgesamt 4 Prozent der Bevölkerung bemerkenswert gering ist insbesondere auch die Gruppe derer, die nicht nur häufigen, sondern auch eher intensiven Ängsten ausgesetzt ist.

\section{Zusammenfassung und Diskussion}

Als Ergebnis dieser Studie zeigt sich auch anhand der Wiener Daten die Differenziertheit des Themas »Angst vor Kriminalität haben«. Die Gruppe der »Ängstlichen « ist nicht homogen »ängstlich«, sondern zerfällt in mehrheitlich »unängstliche Ängstliche « und in eine kleine Gruppe von StadtbewohnerInnen, die als sehr oder permanent ängstlich einzustufen ist.

Dr. Gert Feistritzer, Projektleiter am Institut für empirische Sozialforschung (IFES), Wien; e-mail: gert.feistritzer@ifes.at

\section{Literatur}

Bauer, Christian (1981): Unsicherheit in Wien, Wien (IFES)

Bauman, Zygmunt (2005): Verworfenes Leben. Die Ausgegrenzten der Moderne, Hamburg (Hamburger Edition)

Biderman, Allan, D.et.al. (1967): Report on a Pilot Study in the District of Columbia on Victimization 
and Attitudes Toward Law Enforcement, President`s Commission on Law Enforcement and Administration of Justice, Field Survey I, Washington DC, US Government Printing Office

Boers, Klaus (1991): Kriminalitätsfurcht. Über den Enstehungszusammenhang und die Folgeerscheinungen eines sozialen Problems, Centaurus (Pfaffenweiler)

Bottoms, Anthony, E. (1994): Environmental Criminology, in: Maguire, Mike/Morgan, Rod/Reiner, Robert (eds.), The Oxford Handbook of Criminology, Oxford (Clarendon Press), 585-656

Ditton, Jason / Farrall, Stephen (eds.) (2000): The Fear of Crime, Aldershot (Ashgate)

Farrall, Stephen / Gadd, David (2004): The Frequency of the Fear of Crime, in: British Journal of Crimino$\operatorname{logy} 44,127-132$

Feistritzer, Gert / Raml, Reinhard (2003): Leben und Lebensqualität in Wien, Wien (IFES).

Herrmann, Heike/Sessar, Klaus/Weinrich, Martin (2003): Unsicherheit in der Moderne am Beispiel der Großstadt. Kontexte eines europäischen Forschungsprojektes, in: Stangl, Wolfgang/Hanak, Gerhard (Hrsg.), Innere Sicherheiten. Jahrbuch für Rechts- und Kriminalsoziologie 2002, Baden-Baden (Nomos) 251-286

Hirtenlehner, Helmut / Pilgram, Arno (1999): Sekundäranalyse der Umfragestudie "Leben in Wien«: Wie »sicher« erleben die Bewohner ihre Stadt, Wien (Forschungsbericht des Instituts für Rechts- und Kriminalsoziologie)

InSec (2005): www.irks.at

Mayhew, Pat / van Dijk, Jan J.M. (1997): Criminal Victimisation in Eleven Industrialised Countries; Key Findings from the 1996 International Crime Victim Survey, The Hague

Obergfell-Fuchs, Joachim (2001): Ansätze und Strategien kommunaler kriminalprävention - begleitforschung im Pilotprojekt Kommunale Kriminalprävention in Baden-Württemberg anhand der Stadt Freiburg im Breisgau, Freiburg (edition iuscrim)

Oberwittler, Dietrich / Höfer, Sven (2005): Crime and justice in Germany: An analysis of recent trends and research, in: European Journal of Criminology, vol. 2, nr. 4, 465-508

Stangl, Wolfgang (1996): Die Unwirtlichkeit der Stadt als Bedrohung. «Disorder « und «Crime« in WienMeidling. in: Hammerschick Walter, KarazmanMorawetz Inge, Stangl Wolfgang (Hrsg.): Die Sichere Stadt. Prävention und kommunale Sicherheitspolitik. Jahrbuch für Rechts- und Kriminalsoziologie 1995. Baden-Baden (Nomos), 39-55

Stangl, Wolfgang (1996): «Wien - Sichere Stadt« - Ein bewohnerzentriertes Präventionsprojekt. Kriminologisches Journal, 28, 1996, 48-68

UNICRI (2004): Criminal Victimisation in Urban Europe. Key Findings of the 2000 International Crime Victim Surveys, www.unicri.it/icvs

\section{Anmerkung}

* Zur besseren nationalen Einschätzung der Wiener Ergebnisse sei zunächst auf die Großstudie »Leben und Lebensqualität in Wien (Feistritzer / Raml, 2003) verwiesen, in der gleichfalls ein hohes Sicherheitsempfinden der Wienerinnen und Wiener festgestellt werden konnte. Bezogen auf die eigene Wohnumgebung gaben $73 \%$ der Befragten an, dass sie sich vor Kriminalität (sehr) sicher fühlen; bei $9 \%$ war dies nicht der Fall - es ist dies eine Verteilung, die jener in der Grafik 1 entspricht.

Diese Studienergebnisse fügen sich in Daten aus früheren Befragungen in Wien ein. Bereits in der ersten großen Sicherheitsstudie aus dem Jahr 1981 wurde das weit verbreitete Sicherheitsgefühl in
Wien dokumentiert (Bauer 1981): mehr als 8 von 10 Befragten gaben an, sich beim nächtlichen Spaziergang im Wohngebiet ziemlich oder ganz sicher zu fühlen - ein Ergebnis, das, bezogen auf Österreich, auch in einer Befragung 1996 fast unverändert wieder ausgewiesen wurde (Mayhew / van Dijk 1996; siehe auch Hirtenlehner / Pilgram 1999; FESSEL-GfK 2001).

Der internationale Vergleich belegt die relative Angstfreiheit Wiens in Relation zu anderen Städten. Der Vergleich der Unsicherheitswahrnehmun-

\section{Grafik 6}

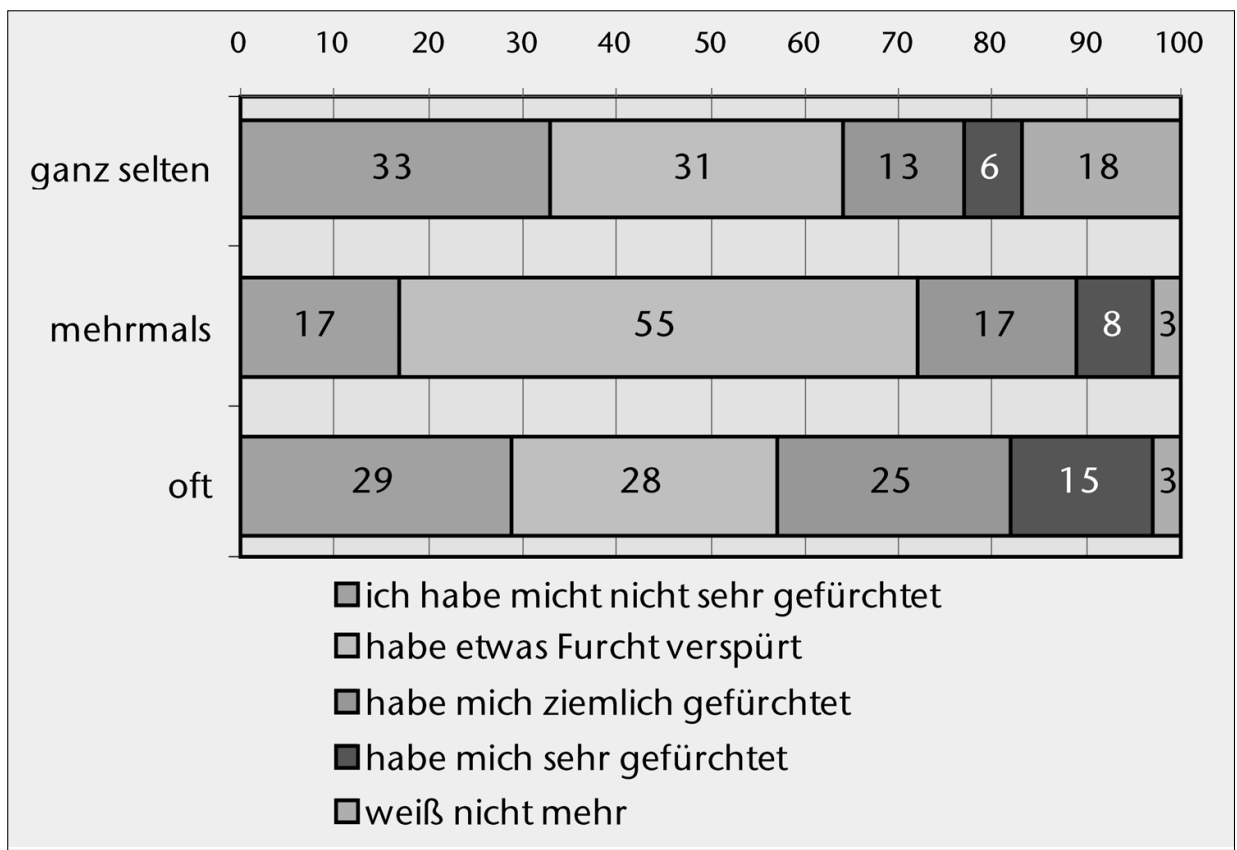

Häufigkeit und Intensität der Kriminalitätsfurcht ( $n=175$; in Prozent)

\section{Grafik 7}

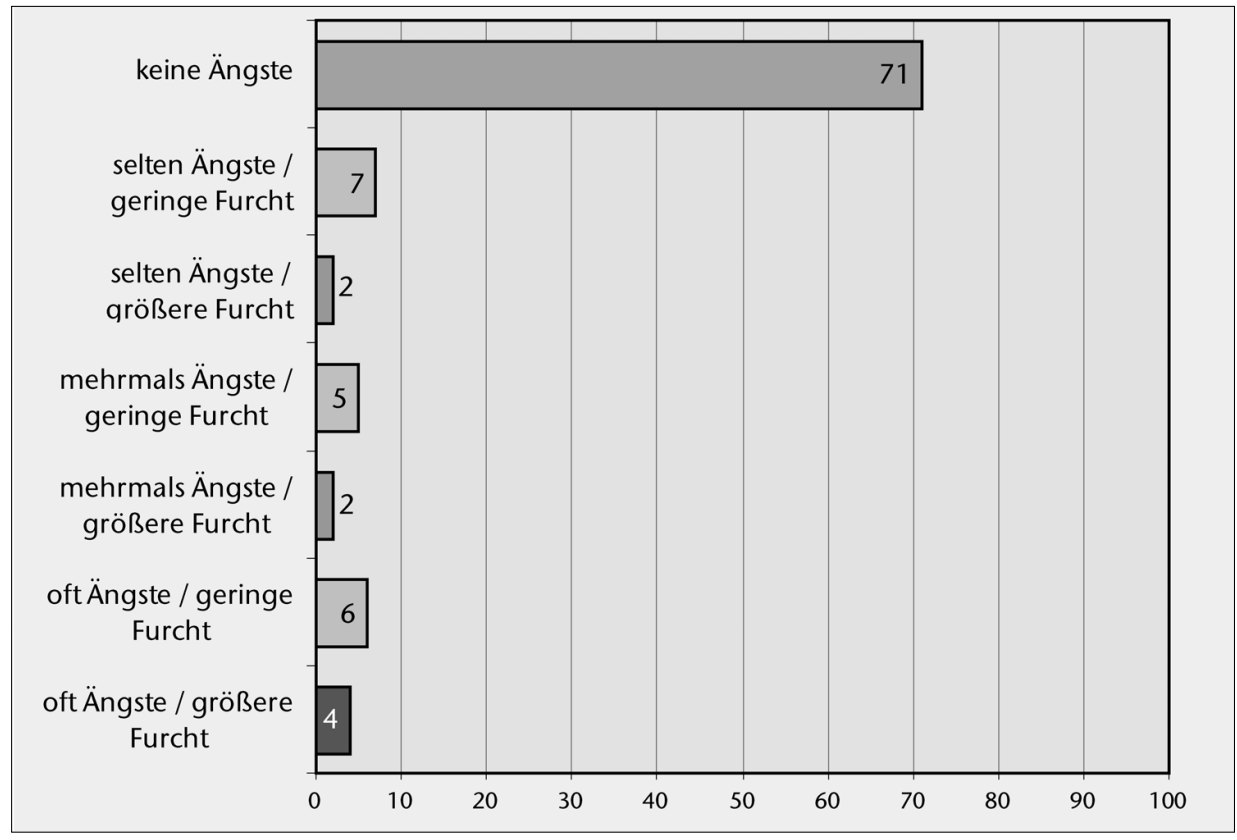

Häufigkeit und Intensität der Kriminalitätsfurcht Basis: Gesamtbevölkerung ( $n=600$; in Prozent) 